\title{
A SPECIAL CASE OF LONGEST PATH PROBLEM
}

\author{
THINH D. NGUYEN
}

\section{DEFINITION AND CLAIM}

\section{Definition 1. Long Path Problem:}

Input: An undirected graph $G=(V, E)$

Output: YES if there exists a path of length at least $\frac{|V|}{4}$ in $G$, otherwise No

Idea: Reduction from Hamiltonian path. Try to think how many vertices should be added to a special instance $\mathcal{G}=(\mathcal{V}, \mathcal{E})$ of longest path problem so that if there is a path of length at least $\frac{|\mathcal{V}|}{4}$ in $\mathcal{G}$ then there is a Hamiltonian path in $G$.

It is easy to see that requiring the graph $G$ to be connected does not decrease the hardness of our problem here. So, in what follows, we always assume (and try to maintain) that $G$ is connected.

Claim 2. We have that Hamiltonian Path $\leq_{p}$ Long Path Problem

\section{Reducing Hamiltonian Path to Long Path Problem}

In this section, we prove the claim 2 .

Proof. Reduce from Hamiltonian Path between 2 specified vertices, namely $s, t \in$ $V(G)$

As mentioned above, we want to add new vertices to the graph while keeping it connected.

Now, do some arithmetics:

- A Hamiltonian path between $s$ and $t$ is of length $n-1$, where $n=|V|$

- If we attach some paths of length (at most) $k$ to $s$ and $t$, then we can increase the length of a path up to $n-1+2 k$.

We should have $4(n-1+2 k)=|\mathcal{V}|$ in the new graph $\mathcal{G}(\mathcal{V}, \mathcal{E})$.

So $|\mathcal{V}|-|V|=3 n-4+8 k$. Take $k=\sqrt{n}$. Do this in an alternative manner, attach to $s$ and then to latext and again to latexs, etc. a new short path of length (at most) $k$ (i.e. $k$ new vertices).

The point of setting $k=\sqrt{n}$ is to prevent one from concatenating 2 short paths attached to the same vertex (either $s$ or $t$ ). So, one must take one path attached to each vertex among $s$ and $t$. And the middle part of the path is a Hamiltonian path between $s$ and $t$.

Key words and phrases. graph, path, hamiltonian.

Perebor. 


\section{Conclusion}

Garey and Johnson [1] shape their theory based on previous primal works of Cook, Levin and Karp. Johnson [2] moves on with the guide to this theory. As long as we study a mathematical conjecture, we should encourage ourselves to have enough labour hours on popular mathematics books like these. Then, reading some articles on theory of computing like [3] is a good practice. Only after that, could we think of the ultimate final for all mathematical sciences.

\section{REFERENCES}

1. Michael R. Garey, David S. Johnson, Computers and Intractability: A Guide to the Theory of NP-Completeness

2. David S. Johnson, The NP-Completeness Column: An Ongoing Guide

3. Phan Dinh Dieu, Le Cong Thanh, Le Tuan Hoa, Average Polynomial Time Complexity of Some NP-Complete Problems, Theor. Comput. Sci. 46(3): pp.219-237 (1986)

Email address: kosmofarmer@yandex.com 